Commun: Communication et organisation

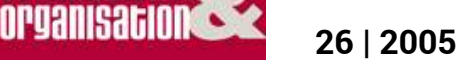

La communication des nouvelles éthiques de l'entreprise

\title{
Les chantiers du manager
}

De Denis Segrestin, Paris, Armand Colin, 2004

Benoit Cordelier

\section{(2) OpenEdition}

Journals

Édition électronique

URL : http://journals.openedition.org/communicationorganisation/3307

DOI : 10.4000/communicationorganisation.3307

ISSN : 1775-3546

Éditeur

Presses universitaires de Bordeaux

Édition imprimée

Date de publication : 2 janvier 2005

Pagination : 271-276

ISSN : 1168-5549

Référence électronique

Benoit Cordelier, "Les chantiers du manager », Communication et organisation [En ligne], 26 | 2005, mis en ligne le 19 juin 2012, consulté le 19 avril 2019. URL : http://journals.openedition.org/

communicationorganisation/3307 ; DOI : 10.4000/communicationorganisation.3307

Ce document a été généré automatiquement le 19 avril 2019

(C) Presses universitaires de Bordeaux 


\section{Les chantiers du manager}

De Denis Segrestin, Paris, Armand Colin, 2004

\section{Benoit Cordelier}

1 Après une douzaine d'années à Grenoble, Denis Segrestin rejoint le Centre de Recherche Innovation Socio-Technique et Organisation industrielle (Sciences Po et CNRS) en 2003. Il nous propose aujourd'hui un ouvrage enraciné dans la pratique managériale, plutôt qu'« un cadre conceptuel "intégré" qui permette de dériver toutes [les] théories $»^{1}$ de l'organisation. Il vise une analyse posée et éclairée des différents mouvements qui traversent la sphère managériale, sans chercher donc d'inutile polémique. Le propos de Denis Segrestin veut en effet se nourrir aussi bien de la littérature "scientifique» que de celle dite de « management » en les nuançant «à l'aide de tous les matériaux empiriques qui [lui ont été] accessibles. ». Après un chapitre liminaire sur la révolution post-taylorienne, il se concentre ici sur sept chantiers « emblématiques de la rhétorique managériale contemporaine ». Nous en donnerons ici un aperçu forcément réducteur.

2 Denis Segrestin rappelle que l'OST a fait l'objet de critiques dès son apparition sans empêcher son avènement en France lors des Trente Glorieuses. La rupture avec la théorie taylorienne s'est faite plus tard, pendant les années quatre-vingt. Cette dernière subissait les premiers chocs d'une mondialisation qui n'allait cesser de s'accélérer. De stratégies centrées sur les coûts de production, les entreprises allaient devoir basculer vers une économie de variété face à un client de plus en plus volatil. Les contraintes d'innovation/ différenciation se font alors plus pressantes. Elles assouplissent continuellement le système productif et amènent l'entreprise à intégrer son organisation (par opposition à la parcellisation taylorienne). Néanmoins, les modèles de changement organisationnel s'hybrident face à la diversité des systèmes de production et des modèles culturels. La stabilité donnant du sens aux comportements, les grandes révolutions annoncées donnent plutôt lieu à des modalités de changement incrémental.

Le premier chantier abordé traite du management des compétences dans la gestion des ressources humaines. Le débat porte sur le passage d'un modèle d'évaluation du travail et du poste associé en termes de qualification ou de compétence. Le premier modèle suppose une qualification prédéterminée et standardisée afin d'occuper des dispositifs de travail 
bien définis. Le deuxième nous amène à considérer la compétence comme une somme de connaissances, de savoir-faire et de comportements structurés en fonction d'un but dans un type donné de situation. La compétence suppose une capacité de coordination horizontale basée sur la pluralité des rôles dans l'organisation. Elle s'appuie donc sur la polyvalence des acteurs, sur un potentiel d'efficacité supérieur aux seuls besoins du poste. Ceci entraîne des difficultés d'évaluation et d'évolution professionnelle et salariale que les managers comme les managés ne sont pas toujours préparés à surmonter. C'est d'autant plus flagrant que le soupçon d'une nouvelle forme d'aliénation du travail transparaît alors: l'autonomie des salariés est encouragée, mais ils en deviennent paradoxalement d'autant plus asservis aux objectifs de la direction. Cette dernière et d'autant plus renforcée dans son autorité que la gestion individualisée, supposée par ce modèle, facilite l'éclatement du collectif des employés. Les syndicats s'opposent logiquement dans un premier temps à ce nouveau modèle, mais ils sont contraints de faire évoluer leur position afin de permettre la reconnaissance et la revalorisation du travail réel des emplois les moins qualifiés. Les rhétoriques du soupçon et du nouveau contrat social évoluent au contact de la réalité (difficulté à établir des indicateurs consistants et consensuels; la réalité des promotions bloque la motivation collective). L'entreprise tend alors à revenir aux logiques de poste et de qualification.

La flexibilité est un autre des enjeux de l'organisation. L'une des façons d'en gagner est de jouer la carte de l'externalisation et du partenariat. Cette décision n'est pas seulement économique, elle est avant tout stratégique et annonce le gouvernement d'entreprise par des contrats externes. Il se réalise, au-delà de la sous-traitance classique, par des relations de partenariat interentreprises. Ces systèmes d'activités distribuées, ces réseaux donnent lieu à des "chaînes de valeur", selon l'expression de Michael Porter. Mais ils n'ont été rendu possibles, non pas par une simple volonté de coopération, mais plutôt par des processus de sélection drastique afin de réduire le nombre de sous-traitants et donc d'intervenants. Le partenariat (ou entreprise étendue) joue à la fois sur la flexibilité du contrat et les atouts de l'intégration. Il apporte, en plus des possibilités de loyauté et de défection, celle non exclusive de négocier tout en restant dans la relation partenariale. Cette dernière crée des synergies sans que la simultanéité des engagements entre partenaires ne soit obligatoire. Néanmoins, au-delà de cette force mythique, l'organisation-marché produit des effets incertains. La variété des dispositifs, les difficultés pour définir le métier du donneur d'ordre et l'appréciation de la distance entre l'entreprise principale et les prestataires périphériques sont autant d'obstacles à sa réalisation. Reprenant Pierre Veltz, Denis Segrestin nous explique que les risques continuent d'exister une fois le partenariat lancé. L'opportunisme local et la rétention d'information peuvent casser cette belle synergie. La flexibilité d'adaptation peut freiner la flexibilité d'anticipation. Le besoin de contrôler ses partenaires peut mener à la saturation des échanges horizontaux par une sollicitation excessive. De plus, l'extrême variété des publics en présence prive les individus de repères identitaires indispensables à la structuration de l'organisation. Un nécessaire compromis passerait alors par l'apparition d'une nouvelle forme de compétence industrielle dans la gestion des interactions partenariales. Enfin, se pose la question du périmètre réel de l'entreprise, notamment dans une dynamique de mondialisation s'appuyant sur des processus de relocalisations multipolaires plutôt que sur des délocalisations (Veltz, 1996).

5 La flexibilité porte également sur le management de la qualité. Celle-ci, depuis le Management de la Qualité Totale (TQM), porte non pas sur les produits, mais sur les 
procédures de l'organisation contrôlée. L'entreprise normalise et enregistre ses procédés sans forcément coller aux meilleures pratiques (best practices) extérieures. Mais la norme subit un procès. Le système est considéré asymétrique, plus favorable au client, et freinant l'innovation. Il favoriserait également les regains d'opportunisme et remettrait en cause les partenariats, même si la certification s'est fixée comme objectif la fluidification des marchés en facilitant les comparaisons. En fait les PME cèdent plutôt au formalisme rhétorique de la norme plus qu'à ses ambitions stratégiques. La véritable opportunité passe par une appropriation et par l'occasion d'un apprentissage collectif continu. De même l'autocontrôle qu'elle véhicule permet son réaménagement par l'employé, puis l'atelier qui se préservent un espace d'initiative. Enfin, si la démarche qualité a pu démarrer sous la pression du client prescripteur, elle a par la suite amélioré le pouvoir de négociation des entreprises l'ayant mise en œuvre et, ayant su se l'approprier. La force de la norme n'est plus ici dans la rigidité, mais dans sa malléabilité.

Le management des processus nous apporte une nouvelle façon de penser l'entreprise à partir des flux à valeur ajoutée. Le Business Process Reengineering (BPR) est une stratégie de rupture critiquée pour les risques de destruction de la culture et des compétences de l'entreprise. Acclamé en son temps par les consultants, c'est une version favorisant une optimisation continue des processus qui a prévalu au travers notamment des Groupes d'Amélioration Continue (GAP). Les processus préoccupaient déjà pourtant les managers : la lean production et sa remise en cause des structures en faveur des flux, l'approche du contrôle de gestion avec la méthode $\mathrm{ABM} / \mathrm{ABC}$, la démarche TQM et ses normes ISO également préoccupées par les flux, la méthode statistique Six Sigma...Les processus ont envahi le discours et l'imaginaire managérial. Leur existence en est d'ailleurs renforcée par la prolifération des applications informatiques. Ils en ont ainsi bousculé les paradigmes de la performance : nous passons ainsi d'une verticalité favorisant le contrôle à une transversalité lui préférant l'analyse stratégique et discréditant par la même occasion les récentes organisations en centre de profit (ou de coût). Le risque est ici d'opposer dans l'imaginaire managérial le flux à l'impératif des bonnes organisations. Mais, même si la complexité de la démarche favorise l'apprentissage organisationnel, la restructuration permanente prive des éventuelles vertus de l'expérience et des routines organisationnelles. De même le pilotage par processus peut entraîner, au profit de décisions de rentabilité immédiate, une déconnexion des décisions stratégiques par rapport à l'environnement et à la communauté productive. Les acteurs de l'entreprise peuvent également se sentir menacés et chercher à se retrancher derrières les frontières fonctionnelles. Un compromis doit par conséquent être trouvé dans les actions quotidiennes de l'action.

7 Cette innovation permanente se retrouve également dans le management de projet qui résulte du rapprochement paradoxal de l'exploration et de la répétition. Il est confié à des équipes sur lesquelles l'organisation s'appuie pour accélérer les rythmes d'incubation de l'innovation. Les équipes projets doivent se projeter en permanence dans l'avenir dans un milieu incertain grâce à un perpétuel échange avec les autres membres de l'équipe aux compétences complémentaires. Leur objectif est, en s'organisant en interne, d'engranger les savoirs tout en retardant les effets de cliquet. Le chef de projet, délégué par la direction générale pour coordonner des échanges à l'issue incertaine, doit faire preuve à la fois d'une autorité charismatique et bureaucratique rationnelle afin de dépasser les logiques fonctionnelles de la structure. Et si l'encadrement par des normes fiabilise le projet et rassure la direction générale, il faut se méfier d'une trop grande formalisation 
qui bloque les capacités d'innovation. Ce risque est d'autant plus prégnant que l'organisation en projet tend à s'inscrire durablement dans l'entreprise dépassant les anciennes frontières, comme la disparition de l'opposition métier/projet. Les nouvelles trajectoires managériales en sont d'autant plus difficiles à anticiper.

L'organisation doit gérer son apprentissage organisationnel par un knowledge management (KM - ou management des connaissances). Sa compétitivité ne passe plus par ses structures et son efficacité fonctionnelle, mais par la gestion de son capital immatériel. Nonaka et Takeuchi revalorisent dans ce contexte l'encadrement intermédiaire qui donne du sens à l'action déterminée par l'encadrement supérieur et qui est concrétisée par les employés. L'organisation s'ouvre et devient une structure "hypertexte». Ces concepts profitent de l'essor des NTIC et en particulier d'Internet. Le groupware, logiciels d'échange collaboratif, combiné par ailleurs à internet, permet le développement de projets et l'inventaire des connaissances accumulées favorisant une veille technologique confiée au nouveau knowledge manager, très vite intégré à la Direction des Systèmes d'Information (DSI). Le KM se répand dans l'entreprise, poussé par la logique d'ouverture qui l'imprègne et par le développement du marché des logiciels. Les outils de coordination, workflow, ne tardent pas à s'engouffrer dans la brèche transportant avec eux une logique de contrôle. L'information foisonne et le KM semble débordé par les possibilités techniques. Comment faire le tri dans ce contexte entre connaissance et information? Le passage du savoir tacite au savoir explicite se fera par le réglage et l'intelligence dans l'organisation, mais non sans difficulté. En effet, le KM peine à se concrétiser et ne finit par réussir qu'au prix d'une subordination aux logiques d'action à l'œuvre sur le terrain. S'il s'en détache, autrement dit si le besoin de coordination ne préexistait pas, les acteurs freineront l'innovation et simuleront l'utilisation des outils pour masquer leur difficulté à partager leur travail avec des interlocuteurs qu'ils ne reconnaissent pas.

9 Les outils, notamment informatique, marquent l'action managériale. Il fallait les intégrer. Les ERP (Enterprise Resource Planning ou Progiciels de Gestion Intégrés) l'ont fait au milieu des années quatre-vingt-dix à la suite des MRP et autres EDI. Tout le monde aurait donc accès à toute l'information. Les performances en sont accrues et, les pratiques de gestion rationalisées et homogénéisées grâce à ces «machines de gestion». Mais l'action des dirigeants risquait de s'en trouver formatée, ce que ne permettait plus la complexification de l'environnement des organisations. Un bras de fer s'engage alors entre les acteurs et les outils. Les investissements justifient-ils la tentation panoptique au détriment des régulations autonomes au sens de J-D. Reynaud? Toutefois l'effort d'intégration de l'outil permet l'apprentissage organisationnel et ouvre par conséquent l'espace d'innovation. L'ERP n'en finit pas d'être paradoxal. Il est en effet à la fois solidaire de la structure traditionnelle et des processus transverses. Il impose également avec un certain succès une rationalisation en dépit de l'efficience du local dans un contexte aléatoire. S'appuyant sur le mythe de l'intégration, il veut nous convaincre de la supériorité du progiciel généraliste sur le logiciel spécialisé en toutes circonstances. Le management même hésite entre la soumission au formalisme et «l'optimisation esthétique » avant de pencher pour la deuxième option. Dans ce dernier cas, l'ERP devient un terrain d'exercice à la topographie largement ouverte jusqu'à la fin du paramétrage. Les projets l'implantant sont même utilisés à des fins stratégiques pour reprendre en main l'organisation. Cette reprise en main annonce un fonctionnement autoritaire visant à redéfinir les compétences et à réaffecter ou réduire les effectifs. Elle se heurte néanmoins à un impératif de réajustements permanents lors de la confrontation de l'outil à l'usage. 
Ceux-ci se traduisent par des compromis et des désaveux partiels. Ces transactions, ces régulations conjointes, ouvrent un autre espace d'apprentissage organisationnel ainsi que le débat rationalisation vs singularité. Après l'enchantement du technique, nous en revenons au stratégique.

Pour ceux qui auraient encore eu un doute, Denis Segrestin nous rappelle ici que le management et l'innovation organisationnelle ne se font pas sur un boulevard déterministe, mais par «cent voies» qui hybrident les différentes approches. Et si nous pouvons regretter quelques absences (Quid du datamining dans le KM? ERP et CRM partagent-ils les mêmes logiques ?...), il nous offre un riche viatique, facile à lire, qui met en perspective différents "chantiers» auxquels nous sommes confrontés dans l'entreprise. Ce livre est d'autant plus précieux que nous avons généralement le nez dans le guidon et que, alors que nous nous croyons écrasés par l'urgence de l'action, nous en oublions de prendre le recul nécessaire pour optimiser nos chances de réussite. Un livre de chantier.

\section{NOTES}

1. J'emprunte ces paroles à Robert $\mathrm{K}$. Merton qui, dans son livre Eléments de théorie et de méthode sociologique (Paris, Armand Colin, 1997 [1957]), nous met en garde vis-à-vis des grandes théories sociologiques intégrées dont les applications pratiques seraient limitées. 